\title{
Avaliação da Aprendizagem em Sistemas Interativos: uma revisão comparativa focada no SBIE, WIE e WAvalia
}

\author{
Valkiria Venancio $^{1,2}$, Roseli de Deus Lopes ${ }^{1}$ \\ ${ }^{1}$ Universidade de São Paulo (USP) - Escola Politécnica - Sistemas Eletrônicos - Núcleo de \\ Aprendizagem, Trabalho e Entretenimento do Laboratório de Sistemas Integráveis \\ Cep - 05508-010 - Cidade Universitária - São Paulo - SP - Brasil \\ ${ }^{2}$ Universidade de São Paulo (USP) - Faculdade de Educação - Departamento de Metodologia do \\ Ensino e Educação Comparada - Grupo de Estudos e Pesquisa em Etnomatemática \\ Cep - 05508-900 - Cidade Universitária - São Paulo - SP - Brasil
}

\begin{abstract}
Here we present a review of articles published in SBIE, WIE and WAvalia, 2008 to 2012, regarding the evaluation and monitoring of learning through digital resources. Highlighted only those articles involving formative assessment, whether in virtual environments and / or face, any level, form or content. In order to observe how the research has been developed to support the evaluation process of student learning. There has been an increasing number of articles on the subject, but still incipient. The evaluation topic is a great challenge for the teaching practice which still has a long way to go.
\end{abstract}

Resumo. Aqui se apresenta uma revisão de artigos publicados no SBIE, WIE e WAvalia, entre os anos de 2008 a 2012, referentes a avaliação e acompanhamento da aprendizagem por meio de recursos digitais. Foram destacados somente os artigos que envolveram avaliação formativa, seja em ambiente virtuais e lou presenciais, voltados a quaisquer nível, modalidade ou conteúdo. Com o objetivo de observar como as pesquisas vem se desenvolvendo para apoiar a avaliação do processo de aprendizagem do aluno, verificou-se um número crescente de artigos sobre o tema, mas ainda incipiente. O tema avaliação é um grande desafio para a prática docente o qual ainda se tem um longo caminho a ser percorrido.

\section{Introdução}

No que diz respeito à avaliação, a grande preocupação encontra-se no fato de se buscar a qualidade no ensino e na aprendizagem. O que implica no aperfeiçoamento e métodos avaliativos e no estabelecimento de diversos critérios.

A avaliação é uma etapa obrigatória do processo educativo, seja em ambientes virtuais ou não, e em busca da qualidade, é necessário identificar competências na interação docente-discente que evidenciem boas práticas como, o incentivo a criticidade, autonomia, curiosidade, condução ética, desenvolvimento de material didático, capacidade de problematizar, provocar interrogações, 
coordenar equipes de trabalho, incentivar participação e coautoria (BEHAR et al., 2010; SILVA et al., 2010).

É igualmente importante diagnosticar desde o início do planejamento, o grau de educabilidade cognitiva do aluno, ou seja, o seu aprender a aprender na Sociedade do Conhecimento (Fonseca, 1998 in ANACLETO et al., 2010). Somente após esse diagnóstico (avaliação diagnóstica) poderão ser definidos conteúdos, estratégias metodológicas, que assegurem o desenvolvimento de competências e habilidades, respeitando o multiculturalismo, realizando o acompanhamento individualizado e avaliando seus processos e progressos (avaliação formativa) e não apenas buscar subsídios nos resultados destes alunos (avaliação somativa) (ANACLETO et al., 2010; VENANCIO e LOPES, 2012).

Behar e outros (2010) apresentam os tipos de avaliação necessários à qualidade educativa, descritos no quadro apresentado em seu artigo (Figura1):

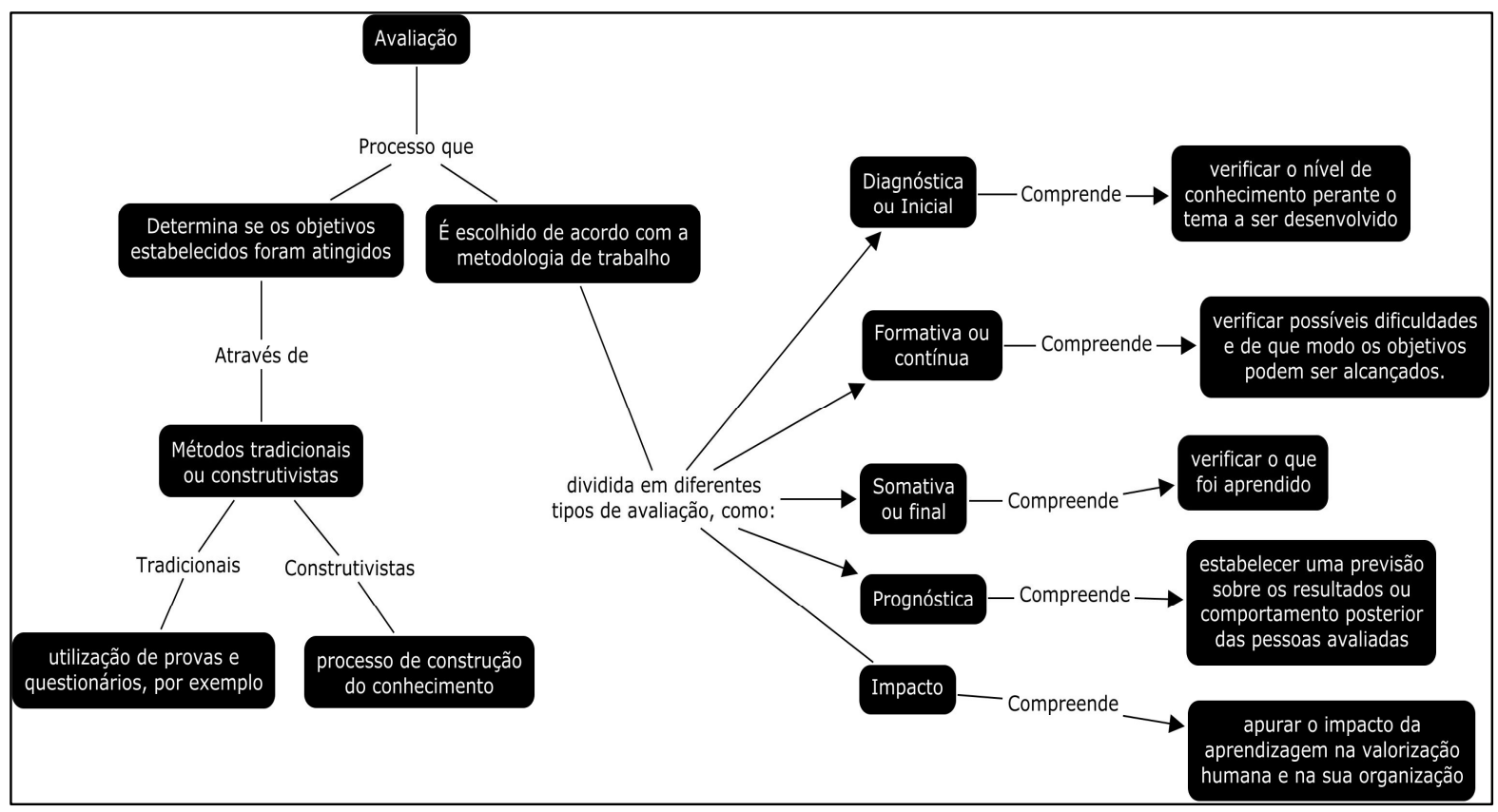

FIGURA 1: Quadro resumo sobre avaliação e formas (BEHAR et al., 2010)

Enfim, seja de forma presencial ou à distância, em qualquer nível de ensino, entende-se que a avaliação deve apoiar e incentivar o processo de construção do conhecimento buscando incessantemente a qualidade no ensino.

A evolução e utilização das novas tecnologias da informação e comunicação "vêm provocando transformações nas concepções de Ciência e impulsionando as pessoas a conviverem com a ideia de aprendizagem vitalícia, sem fronteiras e sem pré-requisitos" (SILVA et al. 2010). O que implica em novas ideias de conhecimento, de ensino e de aprendizagem, e assim, a avaliação apresenta novos propósitos como fornecer realimentação aos estudantes sobre sua aprendizagem, para os professores sobre seus métodos de ensino, para administradores sobre o uso de recursos e para funcionários sobre a qualidade do trabalho desempenhado (SILVA et al., 2010; Borba e Penteado, 2001 in ANACLETO et al., 2010; LEITE et al., 2011; VENANCIO e LOPES, 2012). Neste sentido é fundamental o avanço de pesquisas que busquem associar os benefícios, usos e possibilidades da tecnologia como suporte para a prática pedagógica e otimização do processo de ensino e de aprendizagem. 
A avaliação do processo de ensino e de aprendizagem ainda é um dos maiores desafios da prática docente. Professores e instituições de ensino se deparam com um paradoxo: como avaliar mais alunos e, mesmo assim, dar-lhes um acompanhamento individual e diferenciado? (LEITE et al., 2011; SANTOS et al., 2012).

O uso de ferramentas tecnológicas que forneçam suporte à obtenção e disponibilização de informações relativas ao processo de aprendizagem poderiam auxiliar a enfrentar esses desafios (Bittencourt e Costa, 2011 in GOTTARDO et al., 2012). Entretanto, identificar quais dessas informações são relevantes e manipulá-las de maneira significativa não é um tarefa simples (GOTTARDO et al., 2012). Ferramentas avaliativas associadas aos recursos digitais, adequadas à realidade das instituições e redes educacionais, podem prover ao educador informações importantes sobre o processo de aprendizagem dos seus aprendizes e, baseado nessas informações, ele pode atuar em seus conteúdos a fim de obter um material cada vez mais significativo, e assim, este processo avaliativo pode determinar em que medida os objetivos educacionais estão sendo realmente alcançados (OLIVEIRA et al., 2011; GOMES et al., 2012). As pesquisas e reflexões voltadas à avaliação a partir do uso das tecnologias digitais e suas ferramentas, ainda são incipientes, portanto, o espaço está aberto à realização de pesquisas e produções científicas (LEITE et al., 2011).

Com o intuito de fornecer subsídios às futuras pesquisas, apresenta-se uma revisão de artigos sobre avaliação e acompanhamento do processo de aprendizagem dos alunos com uso de ferramentas digitais. Buscou-se artigos publicados nos Simpósio Brasileiro de Informática na Educação (SBIE), nos Workshop de Informática na Escola (WIE) e nos Workshop sobre Avaliação e Acompanhamento da Aprendizagem em Ambientes Virtuais (WAvalia) no período de 2008 a 2012. Esta revisão traz o seguinte questionamento: como as tecnologias digitais, em ambiente presencial ou virtual, vem se desenvolvendo para apoiar a avaliação formativa, ou seja, a avaliação do processo de aprendizagem do aluno?

\section{Método e Materiais}

Inicialmente foi realizado um levantamento nos anais dos SBIE, dos WIE e dos WAvalia entre os anos de 2008 a 2012, de artigos que dispusessem em seus títulos e resumos termos relacionados à avaliação e acompanhamento do aluno. Independentemente do nível ou a modalidade de ensino ou o ambiente ou conteúdo de ensino, já que o objetivo desta revisão é analisar o desenvolvimento das pesquisas que envolvem tecnologias digitais para o apoio à realização da avaliação formativa da aprendizagem. De um total de 784 artigos publicados nos eventos neste período, foram encontrados 31 artigos sobre o tema nestes cinco anos (Apêndice), ou seja, 3,95\% dos artigos faziam parte deste escopo geral (tabela 1).

TABELA 1: total de artigos publicados no SBIE, WIE e WAvalia de 2008 a 2012

\begin{tabular}{|c|c|c|c|c|c|c|}
\hline EVENTO & $\mathbf{2 0 0 8}$ & $\mathbf{2 0 0 9}$ & $\mathbf{2 0 1 0}$ & $\mathbf{2 0 1 1}$ & $\mathbf{2 0 1 2}$ & TOTAL \\
\hline SBIE & 80 & 108 & 88 & 95 & 129 & 500 \\
\hline WIE & 55 & 37 & 60 & 37 & 48 & 237 \\
\hline WAvalia & - & 10 & 14 & 9 & 14 & 47 \\
\hline & & & & & & $\mathbf{7 8 4}$ \\
\hline
\end{tabular}


Após primeira leitura do total de artigos encontrados, o fato da ferramenta idealizada e descrita nos mesmos estarem direcionadas à avaliação formativa da aprendizagem foi critério único de inclusão para análise.

\subsection{Artigos selecionados}

Os artigos selecionados para análise abrangem os ambientes presenciais e/ou à distância e se tratam unicamente de avaliação formativa, objetivo desta revisão, perfazendo um total de 15 artigos.

A tabela abaixo apresenta o nome da ferramenta digital desenvolvida e descrita nos artigos selecionados, o ano de sua publicação, o ambiente e tipo de avaliação para o qual foi idealizado (Tabela 2).

TABELA 2: Artigos selecionados e características

\begin{tabular}{|c|c|c|c|c|c|c|c|}
\hline & \multirow[b]{2}{*}{ Nome do produto } & \multirow{2}{*}{$\begin{array}{c}\text { Ano da } \\
\text { publica } \\
\text { ção }\end{array}$} & \multicolumn{2}{|c|}{ AMBIENTE } & \multicolumn{3}{|c|}{ AVALIAÇÃO } \\
\hline & & & presencial & a distância & diagnóstica & formativa & somativa \\
\hline 1 & Auto-avaliação & 2008 & & $\mathrm{x}$ & & $\mathrm{x}$ & \\
\hline 2 & ACAvA & 2010 & & $\mathrm{x}$ & & $\mathrm{x}$ & \\
\hline 3 & AVATads & 2010 & $\mathrm{x}$ & $\mathrm{x}$ & & $\mathrm{x}$ & \\
\hline 4 & SMA/AVEAD & 2010 & & $\mathrm{x}$ & & $\mathrm{x}$ & $\mathrm{x}$ \\
\hline 5 & DÓRIS (2010) & 2010 & $\mathrm{x}$ & $\mathrm{x}$ & & $\mathrm{x}$ & \\
\hline 6 & modelo ASIBPq & 2010 & & $\mathrm{x}$ & & $\mathrm{x}$ & \\
\hline 7 & AIED & 2010 & & $\mathrm{x}$ & $\mathrm{x}$ & $\mathrm{x}$ & \\
\hline 8 & Educametria & 2011 & & $\mathrm{x}$ & & $\mathrm{x}$ & \\
\hline 9 & QUIZ do Moodle & 2011 & & $\mathrm{x}$ & & $\mathrm{x}$ & \\
\hline 10 & Predizendo Sucesso & 2012 & $\mathrm{x}$ & $\mathrm{x}$ & & $\mathrm{x}$ & $\mathrm{x}$ \\
\hline 11 & $\begin{array}{l}\text { Corregulação/ } \\
\text { avaliação formadora }\end{array}$ & 2012 & $\mathrm{x}$ & $\mathrm{x}$ & & $\mathrm{x}$ & \\
\hline 12 & Learning Vectors & 2012 & $\mathrm{x}$ & $\mathrm{x}$ & & $\mathrm{x}$ & \\
\hline 13 & EDM em AVA & 2012 & & $\mathrm{x}$ & $\mathrm{x}$ & $\mathrm{x}$ & \\
\hline 14 & GENOME & 2012 & $\mathrm{x}$ & $\mathrm{x}$ & & $\mathrm{x}$ & \\
\hline 15 & SeeAll & 2012 & $\mathrm{x}$ & $\mathrm{x}$ & & $\mathrm{x}$ & \\
\hline
\end{tabular}




\subsection{Descrição dos recursos digitais idealizados e selecionados}

Esta seção se destina a breve descrição dos recursos idealizados ou desenvolvidos pelos pesquisadores selecionados para análise, tal qual disposto nos artigos.

Autoavaliação (2008): experimento no uso da autoavaliação por meio do questionário do Moodle (com modificações) e a ferramenta mensagens. A autoavaliação realizada com frequência apresentou como resultados: mudanças de comportamento, dependência da orientação do tutor e replanejamento de suas ações, melhoria na utilização dos recursos, atendimento individualizado, aumento do número de acessos à própria autoavaliação (PRIMO, 2008).

ACAvA (2010): proposta de avaliação por competências, aplicada via Web na disciplina de Cálculo Diferencial, apoiada no nível de aquisição de conhecimento, mapa conceitual e ontologia (ANACLETO et al, 2010).

AVATads (2010): apresenta parâmetros, regras e resultados utilizando o modelo fuzzi que monitora o avanço ou retrocesso do aluno, como apoio a tomada de decisão do professor (MALVEZZI et al., 2010).

SMA/AVEAD (2010): modelo de sistemas multiagentes centrado na avaliação e estilos de aprendizagem, como apoio a tomada de decisão do professor (LAZZAROTTO et al., 2010).

DÓRIS (2010): exploração inicial no uso do método clínico piagetiano por meio de agente pedagógico tutor animado, um agente este que expressa emoções (SILVA et al., 2010).

Modelo ASIBPq (2010): proposta de avaliação da aprendizagem baseada no sóciointeracionismo. Apresenta o modelo ASIBPq, modelado em redes Petri, para avaliação de fóruns por meio de métricas qualitativas e quantitativas (VASCONCELOS et al., 2010).

AIED (2010): estudo sobre uso de ferramentas avaliativas e mapas conceituais na regulação de conteúdos didáticos. Os resultados do uso do ambiente virtual desenvolvido mostraram que a regulação certa, tanto na avaliação prognóstica quanto na formativa, resulta em conteúdos significativos aos alunos (OLIVEIRA et al., 2010).

Educametria (2011): apresenta uso de modelo de decomposição tensorial Tucker3 para análise de dados (multivariados) da avaliação. É uma categorização qualitativa e quantitativa entre alunos e dimensão da aprendizagem, na decomposição dos dados do componente curricular, metodologia do professor e critérios de avaliação (VASCONCELOS et al., 2011).

QUIZ do Moodle (2011): descrição do uso da ferramenta quiz do Moodle na investigação de sua eficácia e potencial na mediação da aprendizagem e avaliação dinâmica e assistida (LEITE et al., 2011).

Predizendo o sucesso (2012): estudo de caso de uma proposta de diagnóstico da evolução e da identificação dos alunos com dificuldades. Por meio de algoritmos de mineração de dados, geram-se modelos preditivos (SANTOS et al., 2012).

Corregulação e avaliação formadora (2012): descrição de análise de requisitos e concepção de artefato social para regulação de dificuldades de aprendizagem. Propõe a avaliação formadora com cooperação em pares (ROLIM et al., 2012).

Learning Vectors (2012): apresenta um sistema de controle acadêmico e gerenciador da aprendizagem fundamentado em métricas não lineares do Learning Vectors. Implantado no Moodle, atua sobre as interações intermediadas pelas suas ferramentas síncronas e assíncronas (SALES et al., 2012). 
EDM em AVA (2012): investiga a viabilidade da mineração de dados educacionais para a geração de inferências sobre o desempenho de estudantes, como apoio a tomada de decisão do professor, em diferentes etapas do curso (GOTTARDO et al., 2012).

GENOME (2012): ferramenta de avaliação da participação virtual e gerenciamento de notas para o ambiente Moodle (GOMES et al., 2012).

SeeAll (2012): apresenta a implementação de um protótipo de avaliação processual baseado em competências. Sistema de apoio ao planejamento e aprendizagem que organiza e armazena registros sobre vivências de aprendizagem, habilidades, atitudes e competências, retornando aos professores uma visualização em formato de Mapa de Aproveitamento e de Escala de Oportunidades (VENANCIO e LOPES, 2012).

\section{Análise dos recursos digitais idealizados para avaliação formativa}

Observa-se que a avaliação formativa tornou-se uma necessidade na linha história desta revisão, já que no último ano, 2012, mais artigos surgiram nesta linha teórica.

Em breve citação, o sistema educacional brasileiro, desde o final da década de 80 , vem aplicando avaliações em diferentes sistemas estaduais ${ }^{1}$. Avançaram de provas objetivas, que buscavam o diagnóstico das escolas do Brasil, até questões que hoje identificam habilidades específicas, que apontam e recomendam ações em pontos críticos da disciplina para a qual foi aplicada (GATTI, 2002). Anteriormente a tais avaliações, pesquisas brasileiras, baseadas no manual de avaliação de Bloom, Hastings e Madaus $(1971)^{2}$, já refletiam a avaliação formativa em nossas escolas, reflexões que só foram retomadas posteriormente, com grande intensidade, ao serem estabelecidos os Parâmetros Curriculares Nacionais (1990) $)^{3}$. Aparentemente, a evolução da avaliação nos sistemas eletrônicos trilhou os mesmos caminhos, partiu de questões objetivas à preocupação em avaliar o processo de aprendizagem do aluno, mas esta afirmação ainda depende de estudos mais aprofundados.

$\mathrm{Na}$ análise dos recursos observa-se, que mais da metade das pesquisas selecionadas, ou seja, $66,7 \%$, estavam dirigidas ao levantamento de dados e informações sobre a aprendizagem do aluno, de forma a apoiar o professor em sua análise e à sua tomada de decisão, são eles o ACAvA, o AVATads, o SMA/AVEAD, o ASIBPq, o AIED, o Educametria, o Predizendo o sucesso, o Learning vectors, o EDM e o SeeAll. Estes recursos favorecem e respeitam o ensino individualizado e diferenciado, na avaliação de processos e progressos.

Algumas das pesquisas (20\%) apontaram para a tomada de consciência, por professores e alunos, do estado atual da aprendizagem e também para a realimentação ao aluno, são elas Autoavaliação, o Dóris e o Corregulação. Assim, os professores podem reelaborar seu trabalho, redefinindo conteúdos e estratégias, ou os alunos reorganizar seus estudos.

E, por fim, duas das pesquisas $(13,3 \%)$ eram voltadas à simples forma diferenciada de realização da avaliação formativa como descrito no Quiz e no GENOME. O que, de certa forma, incentiva a execução das avaliações e o preparo antecipado pra tal.

\section{1- http://provabrasil.inep.gov.br/historico}

2- Bloom, BS, Hastings, JT and Madaus, GF (1971). "Handbook on Formative and Summative Evaluation of Student Learning". New York: McGraw-Hill. Burnhill, P. and Hartley, J. (1975).

3- http://portal.mec.gov.br/seb/arquivos/pdf/livro01.pdf 
Observa-se que poucas são as ferramentas digitais que devolveram uma visualização da informação e dos resultados de maneira facilitada para que, diante de grande número de alunos, o professor possa fazer uma análise segura e rápida do processo de aprendizagem individualizado, são elas o AIED e o SeeAll. O que demonstra carência na área de visualização da informação, dada a necessidade imprescindível de frequentemente visualizá-los no acompanhamento dos alunos.

Alguns dos recursos podem ser utilizados somente em ambientes virtuais outros estão voltados para ambos, o presencial e o virtual, o que pode contribuir com as unidades educacionais presenciais em diferentes níveis de ensino, não somente à educação a distância.

\section{Considerações Finais}

O processo avaliativo em sistemas educacionais deve compreender o diagnóstico, o processo de aquisição do conhecimento e a produção final, portanto, se entende que as ferramentas destinadas à avaliação devam abranger estas três fases - diagnóstica, formativa e somativa.

No entanto, elaborar um diagnóstico e verificar a aprendizagem final aparenta ser muito mais simples, por se aportar no próprio levantamento de dados, do que avaliar o processo de construção do conhecimento e, se necessário, buscar certa mudança metodológica, devido às diferenças individuais de aquisição da aprendizagem e culturais, motivo pelo qual se apontou esta revisão unicamente para a avaliação formativa.

Ao se pensar recursos digitais para avaliação certamente se deseja aperfeiçoar os já existentes e apoiar o processo de construção do conhecimento com mais qualidade. No entanto, é importante salientar que o criador deve afinar-se com especialistas da área educacional, no intuito de aproximar sua criação da realidade e das necessidades reais de sala de aula.

\section{Referências Bibliográficas}

ANACLETO, G.M.C.; RATOLA, G.B.; OMAR, N. "Avaliação da Aprendizagem em Educação a Distância". Anais Simpósio Brasileiro de Informática na Educação, João Pessoa, PB, 2010.

BEHAR, P.A.; RIBEIRO, A.C.R.; LONGARAY, A.N.C.; BERNARDI, M.; SILVA K.K.A. "AVALEAD: um objeto de aprendizagem sobre avaliação em Educação a Distância". Anais Simpósio Brasileiro de Informática na Educação, João Pessoa, PB, 2010.

GATTI, B. A. “Avaliação educacional no Brasil: Pontuando uma história de ações”. EccoS Rev. Cient., UNINOVE, São Paulo: (n. 1, v. 4): 17-41, jun/2002. Disponível em: http://www.redalyc.org/articulo.oa?id=71540102, acesso em 23/09/2013.

GOMES, A.V.; OLIVEIRA, D.S.da S.; MEDEIROS, A.da C. "GENOME: uma ferramenta avaliativa de participação virtual”. Anais Congresso Brasileiro de Informática na Educação, Rio de Janeiro, RJ, 2012.

GOTTARDO, E.; KAESTNER, C.; NORONHA, R. V. "Previsão de Desempenho de Estudantes em Cursos EAD utilizando Mineração de Dados: uma Estratégia Baseada em Séries Temporais". Anais Congresso Brasileiro de Informática na Educação, Rio de Janeiro, RJ, 2012.

LAZZAROTTO, L.L.; OLIVEIRA, A. de P.; BRAGA, J.L.; PASSOS, F.J.V.; LISBOA Fo, J. “Um Modelo Multiagente para a Avaliação da Aprendizagem em Educação a Distância com Base no Perfil Cognitivo". Anais Simpósio Brasileiro de Informática na Educação, João Pessoa, PB, 2010. 
LEITE, E.A. M.; SALES, G.L.; SOUSA, L. L. R.; JOYE, C. R. “Avaliação Assistida, Feedbacks e Questionários do Moodle”. Anais Simpósio Brasileiro de Informática na Educação, Aracajú, SE, 2011.

MALVEZZI, W.R.; MOURÃO, A.B.; BRESSAN, G. "Uma Ferramenta Baseada em Teoria Fuzzy para o Acompanhamento de Alunos Aplicado ao Modelo de Educação Presencial Mediado por Tecnologia". Anais Simpósio Brasileiro de Informática na Educação, João Pessoa, PB, 2010.

OLIVEIRA, W. P.; SCHIMIGUEL, J.; OLIVEIRA, W. P. de. "Regulação de Conteúdos Didáticos em Ambientes Virtuais de Aprendizagem". Anais Simpósio Brasileiro de Informática na Educação, João Pessoa, PB, 2010.

PRIMO, L. “Auto-Avaliação na Educação a Distância uma alternativa viável”. Anais Simpósio Brasileiro de Informática na Educação, Fortaleza, CE, 2008.

ROLIM, A.L.; GOMES, A.S.; AMORIM, R.; LUCIANO, D. “Artefato Social para avaliação formadora". Anais Congresso Brasileiro de Informática na Educação, Rio de Janeiro, RJ, 2012.

SALES, G.L.; LEITE, E.A.M.; JOYE, C. R. "O Sistema Acadêmico LV: Avaliação em EaD Online Implementada no Ambiente Virtual Moodle”. Anais Congresso Brasileiro de Informática na Educação, Rio de Janeiro, RJ, 2012.

SANTOS, H.L.dos; CAMARGO, F.N.P.; CAMARGO, S.da S. "Predizendo o sucesso de estudantes através do uso avaliações formativas em AVAs". Anais Congresso Brasileiro de Informática na Educação, Rio de Janeiro, RJ, 2012.

SILVA, A.K.; FROZZA, R.; BORIN, M.P.; MOLZ, K.;SCHREIBER, J.; LUX, B.; CARVALHO, A.B.; BAIERLE, J.; KIPPER, L. "O Método Clínico de Piaget como forma de Avaliação da Aprendizagem em um Sistema Tutor Inteligente com Agente Pedagógico". Anais Simpósio Brasileiro de Informática na Educação, João Pessoa, PB, 2010.

VASCONCELOS, F.H.L.; SILVA, T. E. V.; GOMES, P. R. B.; NUNES, A. O.; ANDRIOLA, B.; ALMEIDA, A. L. F. de; MOTA, J. C. M. "Análise do Desempenho Discente em um Ambiente Virtual de Aprendizagem Através de Decomposições Tensoriais Multilineares”. Anais Simpósio Brasileiro de Informática na Educação, Aracajú, SE, 2011.

VASCONCELOS, F.H.L.; NOGUEIRA, O.C.; PRADO, F. Ap.P.; SILVA, T.E.V.; PEQUENO, M.C.; BARROSO, G.C.; MOTA; J.C.M. "Modelagem em Redes de Petri de uma Proposta SócioInteracionista de Avaliação da Aprendizagem em um Ambiente Virtual de Aprendizagem". Anais Simpósio Brasileiro de Informática na Educação, João Pessoa, PB, 2010.

VENANCIO, V.; LOPES, R. de D. "Competências e Avaliação Formativa em Sistema Interativo de Apoio a Aprendizagem e ao Planejamento voltado ao Ensino Fundamental". Anais Congresso Brasileiro de Informática na Educação, WAvalia, Rio de Janeiro, RJ, 2012.

\section{APENNDICE}

Lista de artigos encontrados em primeiro levantamento nos eventos SBIE, WIE e WAvalia entre os anos de 2008 a 2012 e, não referenciados por não fazerem parte do escopo da pesquisa, ou seja, não versarem sobre avaliação formativa. 


\begin{tabular}{|c|c|c|c|}
\hline & Nome do artigo & Autor(es) & $\begin{array}{c}\text { Ano da } \\
\text { publica- } \\
\text { ção }\end{array}$ \\
\hline 1 & $\begin{array}{l}\text { Avaliação docente em ambientes virtuais de } \\
\text { aprendizagem (AVA): propostas de } \\
\text { atividades com o uso do Moodle e Teleduc }\end{array}$ & $\begin{array}{l}\text { DIAS Jr, L.D.; FERREIRA, B. de } \\
\text { J.P. }\end{array}$ & 2008 \\
\hline 2 & $\begin{array}{l}\text { Instrumentação computacional e } \\
\text { realimentação no processo de avaliação para } \\
\text { o ensino de matemática: o conhecimento de } \\
\text { função real como estudo de caso }\end{array}$ & $\begin{array}{l}\text { SOARES, J.M.; BARROSO, N. M. } \\
\text { C.; MOTA, J.C. M.; BORGES } \\
\text { NETO, H. }\end{array}$ & 2008 \\
\hline 3 & $\begin{array}{l}\text { Modelagem em Redes de Petri de uma } \\
\text { Proposta Sócio- Interacionista de Avaliação } \\
\text { da Aprendizagem em um Ambiente Virtual } \\
\text { de Aprendizagem }\end{array}$ & $\begin{array}{l}\text { VASCONCELOS, } \quad \text { F.H.L.; } \\
\text { NOGUEIRA, O.C.; PRADO, F. } \\
\text { Ap.P.; SILVA, T.E.V.; PEQUENO, } \\
\text { M.C.; BARROSO, G.C.; MOTA; } \\
\text { J.C.M. }\end{array}$ & 2008 \\
\hline 4 & $\begin{array}{l}\text { Informática na Educação como apoio à } \\
\text { Avaliação Docente em Disciplina do Curso } \\
\text { de Mestrado em Ciência da Computação da } \\
\text { UFPA }\end{array}$ & $\begin{array}{l}\text { DIAS Jr, L.D.; FERREIRA, B. de } \\
\text { J.P. }\end{array}$ & 2008 \\
\hline 5 & $\begin{array}{l}\text { Uma Ferramenta de Avaliação Automática } \\
\text { para Mapas Conceituais como Auxílio ao } \\
\text { Ensino em Ambientes de Educação a } \\
\text { Distância }\end{array}$ & CALDAS, V. M.; FAVERO, E.L. & 2009 \\
\hline 6 & $\begin{array}{l}\text { Perspectivas metodológicas para o uso de } \\
\text { fóruns em avaliação docente emancipatória }\end{array}$ & $\begin{array}{l}\text { DIAS Jr, L.D.; FERREIRA, B. de } \\
\text { J.P. }\end{array}$ & 2009 \\
\hline 7 & $\begin{array}{l}\text { VIRTUAL-TANEB: Um Ambiente de } \\
\text { Avaliação utilizando Agente de Software e } \\
\text { Ontologia no Processo de Avaliação da } \\
\text { Aprendizagem da Matemática do Quinto } \\
\text { Ano do Ensino Fundamental }\end{array}$ & $\begin{array}{l}\text { BARROS, F.; DINIZ, C.; LABIDI, } \\
\text { S.; COSTA, N.; VERAS, J. }\end{array}$ & 2010 \\
\hline 8 & $\begin{array}{l}\text { Sistema de Previsão de Desempenho de } \\
\text { Alunos para Auxilio a Aprendizagem em } \\
\text { Avaliação de Disciplinas }\end{array}$ & $\begin{array}{l}\text { MAIA, R.F.; } \quad \text { SPINA, } \quad \text { E.M.; } \\
\text { SHIMIZU, S.S. }\end{array}$ & 2010 \\
\hline 9 & $\begin{array}{l}\text { Uso de Lógica Difusa na Avaliação de } \\
\text { Aprendizagem Multidisciplinar }\end{array}$ & WILSON, R.E. & 2011 \\
\hline
\end{tabular}




\begin{tabular}{|l|l|l|c|}
\hline 10 & $\begin{array}{l}\text { Discutindo a avaliação mediada por fóruns } \\
\text { no contexto de cursos totalmente à distância: } \\
\text { uma experiência no Etec-Brasil }\end{array}$ & DIAS Jr, L.D.; COSTA, A.L. & 2011 \\
\hline 11 & $\begin{array}{l}\text { Validação de um modelo de avaliação no uso } \\
\text { da modelagem educacional }\end{array}$ & $\begin{array}{l}\text { LOUZADA, A.N.; ELIA, M. da F.; } \\
\text { SAMPAIO, F. F.; VIDAL, A.L.P.; } \\
\text { RODRIGUES, R. }\end{array}$ & 2011 \\
\hline 12 & $\begin{array}{l}\text { Ferramenta de Identificação de Perfis de } \\
\text { Aprendizes - FIPA }\end{array}$ & BATIVA, G.B.; STIUBIENER, I. & 2011 \\
\hline 13 & $\begin{array}{l}\text { Framework MAAVA- Metodologia de } \\
\text { Avaliação de Ambientes Virtuais de } \\
\text { Aprendizagem }\end{array}$ & $\begin{array}{l}\text { CHAGAS, D.A.; LISBOA, R.P.; } \\
\text { FURO, E.S. }\end{array}$ & 2011 \\
\hline 14 & $\begin{array}{l}\text { Uma proposta de avaliação qualitativa em } \\
\text { ambientes virtuais de aprendizagem }\end{array}$ & $\begin{array}{l}\text { DILLENBURG, D.J.; TEIXEIRA, } \\
\text { A.C. }\end{array}$ & 2011 \\
\hline 15 & $\begin{array}{l}\text { Análise quantitativa do uso das ferramentas } \\
\text { avaliativas dos Ambientes Virtuais de } \\
\text { Aprendizagem e a Taxonomia de Bloom }\end{array}$ & $\begin{array}{l}\text { COSTA, R.D.; LIMA, R. W.; } \\
\text { FERNANDES, R.K. }\end{array}$ & 2012 \\
\hline
\end{tabular}

